\title{
La investigación en Comunicación en Colombia vista desde Web of Science
}

\section{Research in Communication in Colombia seen from Web of Science}

\author{
Arroyave-Cabrera, J., Repiso-Caballero, R. y González-Pardo, R. ${ }^{1}$ \\ Recibido: 20-04-2020 - Aceptado: 25-08-2020 \\ https://doi.org/10.26441/RC19.2-2020-A2
}

RESUMEN: La investigación en comunicación en Colombia es considerada ya un campo consolidado en el contexto nacional, pero no lo es en su proyección internacional. Las bases de datos internacionales y en particular Web of Science se han constituido en una forma extendida de legitimación del conocimiento en la ciencia dentro de Academia Global, pero apenas se ha estudiado la investigación colombiana en comunicación en estos productos. El presente artículo indagó cómo ha sido la producción y circulación de conocimiento en lo que se considera la base de datos más usada a nivel internacional, la Web of Science. Los resultados dan cuenta que la investigación de autores vinculados a la academia colombiana pasó de una figuración muy baja en los años noventa, a un crecimiento importante en 2008 y de manera exponencial en el 2015. La creación de la base de datos Emerging Source Citation Index contribuyó enormemente a este cambio. Del presente análisis se derivan varias implicaciones sobre la producción y apropiación del conocimiento en comunicación en Colombia.

Palabras clave: bases de datos; publicaciones académicas; Web of Science; $\mathrm{ESCl}$; investigación de comunicación en Colombia; capital académico.

\begin{abstract}
Communication research in Colombia is already considered a consolidated field in the national context, but it is not in its international projection. International databases, in particular the Web of Science have become a widespread form of legitimation of knowledge in science at the Global Academy, but Colombian research on communication in these databases has hardly been studied. This article investigated how is the production and circulation of knowledge in one of the most widely used database, Web of Science. The results show that the research of authors linked to the Colombian academy went from a very low figure in the nineties, to a significant growth in 2008 and exponentially in 2015. The creation of the Emerging Source Citation Index database contributed greatly to this change. Implications for the production and appropriation of communication knowledge in Colombia are derived from this analysis.
\end{abstract}

Keywords: databases; academic publications; Web of Science; ESCl; communication research in Colombia; academic capital.

\footnotetext{
${ }^{1}$ Jesús Arroyave-Cabrera es Doctor en Comunicación por la University of Miami (USA), Master en Communication and Information Studies of Rutgers University in New Jersey (USA) y Master en Educación por la Universidad Javeriana-Norte. Es Profesor titular de la Universidad del Norte (Colombia) e Integrante del Grupo de investigación PBX Comunicación, Cultura y Cambio Social. jarroyav@uninorte.edu.co, https://orcid.org/0000-0001-5894-6059

Rafael Repiso-Caballero es Máster en Información y Comunicación Científica por la Universidad de Granada, Doctor en Documentación (2013) y Comunicación (2019). Profesor Titular en la Universidad Internacional de La Rioja. rafael.repiso@gmail.com, https://orcid.org/0000-0002-2803-7505

Rafael González-Pardo es Doctorando en el programa de Comunicación de la Universidad del Norte (Colombia) y Máster en Territorio, Conflicto y Cultura por la Universidad del Tolima (Colombia). Investigador Junior del Departamento Administrativo de Ciencia, Tecnología e Innovación. gonzalezer@uninorte.edu.co, https://orcid.org/00000002-5781-0456
} 


\section{Introducción ${ }^{2}$}

Las primeras investigaciones en comunicación en Colombia, al igual que en América Latina, tuvieron un aporte más centrado en la historia de la comunicación que en la teoría de la comunicación (Barranquero, Arcila \& Arroyave, 2017). Biografías de periodistas famosos, ensayos sobre la libertad de imprenta y escritos relacionados con la regulación legal del periodismo, publicados por lo general en libros, se constituyeron en los primeros aportes autóctonos del naciente campo (Anzola \& Cooper, 1985). Del lento despegue de la investigación a mediados del siglo pasado (1950-1959) se pasaría a la definición de temáticas muy cercanas al contexto nacional, como fueron comunicación y democracia y comunicación y violencia, que dominaron en las agendas de investigación de los años 90 (Martín-Barbero \& Rey, 1999), hasta llegar en el siglo XXI a lo que Pereira (2018) denomina "Etapa de Consolidación". En efecto, la investigación se ha institucionalizado y en la actualidad existe una amplia producción intelectual, la mayoría en forma de artículos científicos. Sin duda la gradual masificación en los programas de comunicación social-periodismo que se dio a partir de los años 70 permitió el surgimiento de una masa crítica de profesores y estudiantes del campo, que sentaron las bases para que floreciera la investigación de manera más estructurada en el país.

No obstante, a pesar de este avance, en su balance de la producción de conocimiento en comunicación en Colombia en el nuevo siglo, Pereira (2018) plantea que uno de los grandes retos es la participación en el concierto internacional. Tanto Anzola \& Cooper (1985) como Martín-Barbero \& Rey (1999) daban cuenta de una amplia producción bibliográfica basada fundamentalmente en libros. Si bien la publicación de libros y capítulos de libros continúa siendo la importante en Colombia, la extensión de un nuevo modelo de legitimación del conocimiento en las ciencias ha traído como consecuencia que se generalicen nuevas dinámicas, donde el artículo de investigación pasó a tener un mayor reconocimiento (Beigel, 2014). Por este motivo, ha aumentado la demanda por publicar en revistas indexadas en bases de datos tanto nacionales como las internacionales, siendo Web of Science (WoS) y Scopus las más valoradas. No solamente los académicos aspiran a publicar en estas revistas, sino que también las revistas vinculadas a instituciones nacionales aspiran a ser indexadas en estas bases de datos (Fischman, Alperin \& Willinsky, 2010). Recientes revisiones sobre la investigación colombiana han incorporado como criterio de análisis y reflexión la producción publicada en revista indexadas a nivel nacional o internacional (Roncallo, Uribe \& Calderón 2013; Valderrama, 2009, 2011).

En Colombia, el Consejo Nacional de la Ciencia y la Tecnología-Colciencias (Minciencias desde el 2019), fiel a esta tendencia mundial, reestructuró los criterios para clasificar a los investigadores en el contexto nacional. Las publicaciones en las revistas indexadas en WoS y en Scopus se consolidaron como las aportaciones con mayor puntuación en la evaluación de la producción intelectual de los investigadores. De igual manera, los libros y/o capítulos de libros con mayor puntuación son aquellos vinculados al Book Citation Index (Colciencias, 2018). Este sistema evaluativo se refuerza porque paralelamente muchas instituciones académicas han condicionado la vinculación y avance del profesor en la escala docente en función no necesariamente de la calidad de la investigación o del impacto en su campo de estudio, sino en la publicación en revistas indexadas en las bases de datos WoS y Scopus (San Fabian, 2020).

Desde su creación en 1951 en Filadelfia, la base de datos bibliográfica llamada en principio ISI

\footnotetext{
${ }^{2}$ El trabajo realizado por Rafael González-Pardo en este artículo hace parte del proyecto "Abordajes del pensamiento comunicacional latinoamericano en revistas científicas", financiado con recursos del Fondo Nacional de Financiamiento para la Ciencia, la Tecnología e Innovación (FCTel) del Sistema General de Regalías (SGR).

Nota: Los autores desean expresar su agradecimiento a Enrique Uribe Jongblond y Adelina Alabarce así como a los revisores anónimos del manuscrito quienes realizaron sugerencias valiosas al trabajo original.
} 
(por su sigla Institute of Scientific Information) y conocida hoy como Web of Science (WoS) se ha posicionado en el principal referente de evaluación de revistas y artículos, convirtiéndose en un monopolio. Instituciones de gran relevancia en el campo de la educación, ciencia y tecnología como la Organización de las Naciones Unidas para la Educación, la Ciencia y la Cultura, - UNESCO, el Science and Technology Observatory-OST (Francia) o The Royal Society- RS (Reino Unido) la utilizan como parámetro para establecer los aportes de los diferentes países y centros académicos en los avances en la ciencia y el conocimiento científico. De hecho, los Informes UNESCO sobre la Ciencia $(2005,2010,2015)$ y el de OST (2019) Dynamics of scientific production in the world, in Europe and in France, 2000-2016, se basan exclusivamente en la base de datos WoS.

A pesar de su trascendencia en el contexto internacional, poco es lo que se ha estudiado en Colombia sobre la investigación en el campo de la comunicación en la base de datos Web of Science. El objetivo del presente artículo consiste en llenar ese vacío y explorar cómo ha sido la producción y circulación de conocimiento en lo que se considera la base de datos más usada a nivel internacional. Además, queremos estudiar a través de la coautoría el capital relacional internacional y su impacto para las instituciones colombianas.

La siguiente sección ofrecerá una contextualización del sistema investigativo en la academia internacional. Luego revisará el surgimiento de los sistemas de medición y su papel en el avance y circulación del conocimiento. Posteriormente se describirá el marco teórico que sustenta esta investigación. De aquí se desprenderán las diversas preguntas de investigación e hipótesis que guían este estudio.

\section{La universidad como fuente de conocimiento y la investigación como fuente de supervivencia}

La universidad en América Latina surgió en la época de los virreinatos españoles y tenía en principio un carácter fuertemente confesional, a imagen de la universidad medieval europea (Alejo Montes, 2007). Era muy común ver más graduados de teología que de medicina. Las universidades en el Nuevo Mundo no fueron en principio muy diferentes a las europeas y muchas de las grandes investigaciones que se dieron en el continente eran lideradas por científicos y sabios que estaban afuera de la universidad, en forma de expediciones científicas (Weinberg, 1997). En el contexto norteamericano no hubo investigación en las instituciones educativas de la colonia. La investigación era promovida por sociedades privadas, museos o naturalistas independientes (Thelin, 2004). De acuerdo con Rogers (1994), los tres personajes que más han influido en el pensamiento social del siglo XIX fueron Charles Darwin, Sigmund Freud y Karl Marx. Ninguno de ellos tenía una vinculación universitaria.

El concepto moderno de la universidad como centro de investigación surgió en Alemania, en el siglo XIX, cuando Wilhelm von Humboldt se estableció como rector en la Universidad de Berlín. Para von Humboldt la universidad estaba llamada a fortalecer el desarrollo nacional y científico del país con el apoyo del Estado. "El concepto humboldtiano demostró ser altamente exitoso, y las nuevas universidades alemanas (y otras que fueron reformadas para conformar el nuevo modelo) contribuyeron al surgimiento de Alemania como un país moderno al producir investigación y educar a científicos" (Albacht 2007, p. 114). La idea del "Herr Professor", que no solamente enseña, sino que sobre todo investiga y con el conocimiento nuevo se convierte en el faro que ilumina con su luz a la sociedad fue un fenómeno en principio alemán y después copiados por otros sistemas como el anglosajón.

El sistema de tenure norteamericano y los sistemas de evaluación europeos (habilitación, acreditación) se basan en el sistema de publish or perish (pública o perece). En efecto, la posibilidad de 
obtener una plaza como profesor con permanencia o tenure (denominado Profesor Asociado) depende en buena medida de la producción científica. Si bien la docencia y la administración tienen importancia, es el número y la calidad de las publicaciones aquello que determina su permanencia en el sistema. Tal como lo afirma Zdenek (2018), la condición más importante para el éxito profesional relacionado con la vinculación y promoción en la academia se basa en las publicaciones en las principales revistas con revisión de pares.

En el contexto europeo o norteamericano, una vez el aspirante a profesor se gradúa de doctor, entra en el sistema en calidad de Profesor Asistente (Assistant Professor) o asistente de investigación. Empieza entonces un periodo de prueba que sirve para que el profesor/investigador junior demuestre que es capaz de asumir una investigación independiente, sin mentor como es su tesis doctoral y que puede realizar aportes originales en su campo de estudio. Transcurrido un cierto tiempo, el profesor es evaluado para, en principio conseguir la plaza en la institución (tenure o permanencia) y luego, para ir avanzando en los diferentes escalafones de la carrera académica. Es aquí donde las principales revistas indexadas cumplen un rol fundamental. Cada universidad establece ciertas expectativas sobre el número de publicaciones que esperan de su profesor. Las universidades de investigación más prestigiosas establecen como parámetros 2 artículos o más en revistas indexadas al año. Otro camino pueden ser los libros o capítulos de libro, pero se espera que sigan un proceso riguroso de revisión de pares y estén vinculados a editoriales académicas prestigiosas. No obstante, a pesar de que este modelo académico se ha extendido por el mundo, no de manera igual las condiciones y ventajas con que puede contar un profesor vinculado a un sistema del Norte Global para efectuar sus investigaciones.

\section{Mirada teórica: buscando sentido a este fenómeno}

Las reflexiones teóricas del sociólogo francés Pierre Bourdieu resultan pertinentes para posar una mirada analítica sobre el sistema académico. Bourdieu $(1988,1998,2004)$ ha planteado que la ciencia es un "juego" cuyos protagonistas son los científicos, que siguen unas reglas claramente prestablecidas. Aquellos que internalizan dichas reglas y las convierten en habitus, es decir parte de su esencia, ganan mayor capital académico "mientras que aquellos menos prósperos pueden sufrir serias desventajas" (Demeter 2019, p. 578).

Dado el carácter de juego del campo académico, cada participante trata de competir para acrecentar su capital. Sin embargo, existen campos de fuerza con reglas claramente establecidas que nos indican cómo se gana ese capital. Los rankings de diferentes naturalezas (instituciones, revistas científicas, casas editoriales, bases de datos) nos dan claras pistas de qué es lo que debemos buscar si queremos ganar o acrecentar ese capital (Bourdieu, 1998). No es de extrañar entonces que quienes tienen mayor capital establezcan las reglas del juego para de esta manera seguir acumulando capital. En este sentido, las mediciones de publicaciones e índices de impacto son concebidas como estadísticas 'universales', mientras son construidas en los centros académicos que están en la cúspide y definen qué es lo 'publicable' (Beigel, 2013; Ortiz, 2009).

En este punto, es pertinente traer a colación otro modelo teórico muy usado para comprender las dinámicas que se dan en la geopolítica del conocimiento a nivel global: el modelo teórico de la dependencia (Beigel, 2013; Demeter, 2019). Si bien la teoría de la dependencia surgió a finales de los años 60 como un intento de reconceptualizar el subdesarrollo a partir de unas nuevas bases que tuvieran en cuenta el sistema-mundo y las circunstancias particulares de los países coloniales, el concepto se ha extendido hoy en día al campo de la educación y se ha acuñado el término de dependencia académica. La dependencia académica se refiere a la estructura desigual de producción y circulación de conocimiento a nivel mundial (Beigel, 2013). 
Centro y periferia coexisten, sin embargo, el poder, el posicionamiento y el dominio de los recursos simbólicos y materiales del centro establecen las reglas del juego y en ese ejercicio, garantiza su dominio. El centro, conformado por los países del Norte Global (NG) ha perpetuado una hegemonía en el campo de la producción de conocimiento en las ciencias en general (Demeter, 2018; Mosbah-Natanson \& Gingras, 2013). Las reglas del juego han sido impuestas en buena medida por el NG, aunque cuestionadas y retadas con mucha frecuencia por el Sur Global (SG) (Chakravartty, Kuo, Grubbs, \& McIlwain, 2018; Mignolo, 2010). Tal como lo ha planteado Bourdieu (1988), quienes tienen mayor capital establecen las reglas del juego, para seguir acumulando capital y perpetuar cierto dominio en el juego.

En efecto, no es exagerado afirmar que la producción de conocimiento no solo en el campo de la comunicación, sino en general en las ciencias sociales es asimétrica, arbitraria y diseñada para perpetuar la hegemonía del Norte Global (NG), (Beigel, 2013, 2016; Mosbah-Natanson \& Gingras, 2013; Demeter, 2019). A efectos de poder producir conocimiento y de esta manera ganar capital debemos internalizar las reglas del juego que nos indican la estructura textual que deben tener los productos intelectuales, el idioma en que debe ser publicado, los procedimientos metodológicos que son válidos para tener cabida en aquellos espacios del campo que la normativa indica como válidos para ganar capital. Quienes ya cuentan con un capital y ocupan posiciones de poder tales como editores de revistas, miembros de comités científicos de una revista o editores de sellos editoriales de alto nivel han incorporado este hábitus y no dan paso a textos que tienen otras perspectivas epistemológicas, otros autores u otros abordajes, igual de rigurosos y que quizás podrían aportar, pero que se salen del canon ya establecido (reglas del juego) por el NG dominante (Albuquerque et al., 2020; Ganter \& Ortega, 2019; Goyanes, 2019).

Diversos estudios arrojan suficiente evidencia empírica que sustenta estas afirmaciones. El exhaustivo estudio realizado por Mosbah-Natanson y Gingras, (2013) de los 200 journals o revistas científicas mejor posicionadas en la base de datos Social Science Citation Index (SSCI) de WoS en el área de ciencias sociales, pone en evidencia como los países del NG obviaron la producción de conocimiento del SG. En efecto, la citación de autores vinculados a la academia latinoamericana por parte de investigadores vinculados a la academia europea y norteamericana en los últimos 30 años es porcentualmente 0. Igual ocurre para continentes como África, Asía, Oceanía y los países del este europeo. Mientras que los autores de la academia norteamericana citan a sus paisanos de manera abrumadora (1983-5: 82.9\%, 1993-5: 80.2\% y 2003-5: 76.7\%), los únicos otros que consideran dignos de leer y citar son los autores de la academia europea (1893-5: 15.8 \%, 1993-5: 18.3 $\%$ y 2003-5: 21.9\%), aunque en menor proporción. En otras palabras, en las revistas internacionales en ciencias sociales de mayor prestigio los autores vinculados al contexto norteamericano citaron en promedio un $80 \%$ de autores del mismo país y un $19 \%$ de autores europeos, desconociendo la producción de conocimiento que se produjo en el resto del mundo.

Al centrar el foco de atención en el campo de la comunicación, Demeter (2018) refuerza la idea de que el aporte desde el SG y en particular de América del Sur sigue siendo muy marginal. El estudio exploró las publicaciones de las 79 revistas en comunicación y estudios de medios indexadas en la base de datos WoS en un período que va de 1975 a 2017. En los 42 años de análisis del estudio, la contribución de América del Sur a estas publicaciones indexadas a Web of Science (WOS) es del $1 \%$. Al hacer un comparativo global se observa que las publicaciones provienen en un $96 \%$ del NG y un $4 \%$ del SG. Hecho que resulta paradójico si tenemos en cuenta que América Latina aporta un poco más del $8 \%$ de la población mundial y que buena parte del continente solo contribuye con una cifra marginal de un $1 \%$.

No obstante, así como Bourdieu (2004) plantea que existen campos de fuerza existen también campos de lucha, en donde distintos agentes poseedores de diferentes recursos se confrontan unos 
a otros para conservar o transformar las relaciones de poder existentes (p. 34). Un ejemplo lo encontramos en América Latina con el denominado Giro decolonial, que cuestiona la dependencia del modelo anglo-europeo como el único supuesto válido para producir el conocimiento (Mignolo, 2010). Así mismo, ha habido un contra-movimiento que busca la des-occidentalización del conocimiento (Downing, 1996; Waisbord \& Mellado, 2014; Hanitzsch, 2019) y que cuestiona las publicaciones "demasiado blancas" (Chakravartty et al., 2018; Ng, White \& Saha, 2020) que han prevalecido en la comunicación por mucho tiempo.

Otros ejemplos de las tensiones que se dan en el campo de lucha provienen de los distintos cuestionamientos a la hegemonía impuesta por estos sistemas de medición internacional. Diversas voces han planteado la tiranía del ranking (Post et al., 2013) y la tiranía del factor de impacto (Palomero Pescador, 2010; Ready, 2014), que establecen las agencias internacionales, que desconocen las realidades particulares en la producción de conocimiento como se dan en los diferentes países. Esta presión internacional, el movimiento de Open Access, y el surgimiento de otras bases de datos (Latindex, Dialnet, DOAJ) ha llevado a las empresas y organizaciones editoriales hegemónicas a ampliar su sistema de indexación (Funes, Heredia, \& Suárez 2011). En el 2015, WoS creó una nueva base de datos denominada Emergin Sources Citation Index (ESCI), que incluye a todas aquellas publicaciones candidatas a alcanzar los índices de la core collection o colección de publicaciones centrales.

La inclusión de estas nuevas revistas en la base de datos WoS, algunas de ellas en idiomas tales como el alemán, español y el chino, al igual que el surgimiento de nuevas revistas en diferentes campos ha permitido que el número de publicaciones a nivel mundial se duplique en este siglo (Science and Technology Obsrevatory-OST 2019, p. 22). De esta manera hemos pasado de 850 mil artículos en el año 2000 a un poco más de 1,9 millones en el 2016. De igual manera, el Reporte de Ciencia de la UNESCO (2015) plantea que el número de artículos publicados por autores latinoamericanos "en los journals catalogados en Science Citation Index Extended se ha incrementado en un $90 \%$ del 2005 al 2014, permitiendo un porcentaje global del 4.0\% al 5.2 \%" (p. 188).

En este estado actual de las cosas, surge un primer tema que queremos indagar. Es de esperarse que la producción intelectual de instituciones colombianas haya mantenido una producción baja en la base de datos WoS. Sin embargo, al dar paso a nuevas revistas en la indexación ESCI, es de esperarse que el número de publicaciones haya crecido en forma amplia.

¿Habrá un mayor número de publicaciones de instituciones colombianas en WoS a partir del nuevo sistema de indexación ESCI?

El capital social, como plantea Pierre Bourdieu (1988), es desde donde se mide la colaboración social entre los diferentes grupos de un colectivo humano, y desde el que hoy las instituciones educativas generan redes sociales entre los diversos actores que confluyen en ella. Como plantean Flores, Palavecino y Montejano (2013), la universidad en su día a día establece cada vez más constantes y variadas relaciones con diversos grupos de agentes, de los que se derivan un conjunto de conocimientos que integran a las instituciones con los diversos públicos que forman parte de ellas, logrando así la generación de capital relacional, dimensiones cada vez más imprescindibles para las instituciones educativas superiores.

El Análisis de Redes Sociales estudia el capital relacional, entendido como el "conjunto de métodos para estudiar los aspectos relacionales de las estructuras sociales" (Scott, 1992, p. 96). Éstas se verifican a través de técnicas centradas en sacar a la luz los patrones de interacción entre las personas (Freeman, 2006). Sanz y Menéndez, (2003) indican que el análisis de redes sociales es "una aproximación intelectual amplia para identificar las estructuras sociales que emergen de las diversas formas de relación, pero también es un conjunto de métodos y técnicas” (p. 21). 
Para esta investigación, se entiende entonces que son estas redes sociales las que permiten a las universidades consolidar sus capitales relacionales, que cada vez más se fortalecen desde escenarios internacionales, y que para el mundo de la producción de las publicaciones científicas, surgen como aquellas redes de colaboración y coautorías entre investigadores. Estas redes son hoy una fuente medible de capital académico (Coccia \& Bozeman, 2016) y desde la que se generan mayores posibilidades de publicación en revistas de alta indexación (Demeter, 2019).

En el contexto colombiano, varias universidades han hecho apuestas concretas para estar a tono con estas reglas de juego y tener mayor participación en redes internacionales de investigación y en proyectos de investigación comparativos. Así mismo, han asumido un liderazgo nacional en la oferta de postgrado al más alto nivel (doctorado) que, a la postre, les ha permitido consolidar una red internacional de investigación. Como sabemos, la formación doctoral trasciende las fronteras nacionales y demanda una interlocución importante a nivel horizontal con la academia internacional.

Mientras la lucha de fuerza en el campo de las publicaciones se consolida y el SG se posiciona con mayor peso, las redes internacionales son esenciales para tener cabida en el sistema. Una amplia investigación sobre el tema respalda la idea que en la investigación actual la única posibilidad para autores de países en vía de desarrollo de convertirse en investigadores internacionalmente reconocidos es inmigrar o cooperar con autores de países desarrollados (Fernández, Ferrandiz, \& León, 2016; Schomoch \& Schubert, 2008; Teodorescu \& Tudores, 2011). Está claro que debe ser en el marco de una colaboración simétrica donde intercambio y diálogo de saberes se dé por igual. Sin duda, el SG tiene que dar pasos para romper la asimetría que existe en las publicaciones internacionales y tener mayor presencia con sus temáticas, epistemologías y tradiciones investigativas en las principales revistas del campo. Demeter (2019) ha planteado que tanto las redes internacionales como la indexación de revistas de SG en las bases de datos dominantes son estrategias importantes para garantizar una mayor visibilidad.

De este aparte se desprenden las siguientes hipótesis:

H.1. Aquellas universidades con una oferta de postgrado completa tendrán más publicaciones en WoS.

H.2. Aquellas instituciones con mayor capital relacional internacional tendrán mayor presencia en la base WoS.

\section{Metodología}

Para el presente estudio se analizaron un total de 191 trabajos (artículos y revisiones) de la base de datos Web of Science (Core Collections) publicados hasta el año 2018. Concretamente 115 artículos en Emerging Source Citation Index, 69 en Social Science Citation Index y 7 artículos en Arts \& Humanities Citation Index en las categorías "Communication" y "Film, Radio \& Television", artículos donde al menos uno de los firmantes incluye una filiación colombiana. Con esta selección quedaron excluidos aquellos trabajos de profesores de investigación colombianos pero que han sido publicados en categorías de WoS que no pertenecen a comunicación. De igual manera, se registraron los trabajos publicados por profesores colombianos de otras disciplinas, pero en revistas de comunicación.

Si bien existen diversas bases de datos de revistas en países iberoamericanos como Latindex, Dialnet (Difusión de Alertas en la Red), DOAJ (Directory of Open Access Journals), REDIB (La Red Iberoamericana de Innovación y Conocimiento Científico) o MIAR (Matriz de Información para el Análisis de Revistas) que han emergido como respuesta al domino de los 
sistemas hegemónicos mundiales de publicación, y aparecen como propuestas alternativas a las bases de indexación referenciales (González-Pardo, Repiso \& Arroyave, 2020). Para la presente investigación se escoge WoS que, a diferencia de estas bases de datos, indexa y clasifica los resultados de sus colecciones a partir de las citas y referencias de las publicaciones científicas que allí se registran, y por lo tanto permite una sistematización, exportación y análisis de los datos que reporta. Por lo tanto, se tienen en cuenta únicamente las revistas referentes en Ciencias Sociales y Humanidades respectivamente: Social Science Citation Index - SSCI y Arts \& Humanities Citation Index $-A H C I$.

Se incluye también en el estudio Emerging Source Citation Index -ESCI, que si bien es una base de datos de segundo orden en relación al impacto de las revistas y sus trabajos, es el producto de Clarivate Analytics que incluye el conjunto de revistas que podrían entrar en las bases de datos preferentes, y a la vez aumentar la cobertura de trabajos de la base de datos de WoS (Repiso y Torres-Salinas, 2016).

Este trabajo analiza los siguientes aspectos:

Tabla 1. Variables y criterios metodológicos.

\begin{tabular}{|ll|}
$\begin{array}{l}\text { Distribución temporal } \\
\text { de la producción }\end{array}$ & $\begin{array}{l}\text { Se realizó una distribución temporal (anual) del número de trabajos publicados en } \\
\text { Web of Science por instituciones colombianas. (Figura1). }\end{array}$ \\
\hline $\begin{array}{l}\text { Distribución por } \\
\text { revistas }\end{array}$ & $\begin{array}{l}\text { Se analizó la concentración de trabajos publicados por instituciones colombianas } \\
\text { de revistas (al menos tres trabajos). Se distinguió la indexación de las revistas } \\
\text { (SSCl, ESCl o A\&HCI) (Figura 2). }\end{array}$ \\
\hline $\begin{array}{l}\text { Se identificaron } 25 \text { centros productores de trabajos de investigación sobre } \\
\text { Comunicación en WoS. Se realizó una tabla de producción (artículos según } \\
\text { presencia en ESCl y SSCl). Métricas de impacto; citas y citas promedio. Métricas } \\
\text { Relativizadas a la categoría "Communication". Impacto Normalizado a la } \\
\text { Categoría y Percentil. Los datos relativizados se obtuvieron en InCites. Se realizó } \\
\text { por centros } \\
\text { un "mapa" de calor para identificar de manera relativizada la posición de los } \\
\text { centros de investigación por cada valor. (Tabla 2). }\end{array}$ \\
\hline $\begin{array}{l}\text { Se estudió cómo las universidades colaboraban entres sí en la producción } \\
\text { de artículos científicos de Comunicación. Los datos han sido procesados con } \\
\text { Red de colaboración } \\
\text { entre instituciones } \\
\text { torno a las co-citas según el algoritmo Louvain (Blondel et al., 2008) se vectorizó } \\
\text { el tamaño de los nodos en relación al número de trabajos en colaboración. Los } \\
\text { centros de investigación no colombianos fueron señalados en color violeta. } \\
\text { (Figura 3). }\end{array}$ \\
\hline
\end{tabular}

Fuente: elaboración propia

El Impacto Normalizado a nivel de artículo es una métrica que permite relativizar de manera individual o agrupada la repercusión medida en citas de un trabajo en relación con el resto de los trabajos de su área en el año de su publicación (Torres-Salinas et al., 2018). En el trabajo actual se ha comparado el impacto que cada trabajo recibe con el impacto promedio del resto de trabajos del área de comunicación que se publicaron ese mismo año en Social Science Citation Index y Emerging Source Citation Index. Posteriormente, se indica la posición que ocuparía el trabajo según su impacto en forma de percentil.

\section{Resultados}

En relación con nuestra primera pregunta de investigación encontramos que siguiendo la tendencia mundial, el número de publicaciones se incrementa de manera significativa cuando WoS incorpora nuevas revistas en la base de datos ESCI. La presencia de investigaciones colombianas en Web of Science comienza en el año 1993 con un artículo de Jesús Martín 
Barbero titulado "Latin-america-cultures in the communication media", publicado en la Journal of Communication. Desde ese primer documento hasta el año 2008 sólo se publican cuatro trabajos (Figura 1). Hasta el año 2008 la presencia de trabajos de autores vinculados a la academia colombiana es mínima, posteriormente y hasta 2015 se muestra cómo todos los años se indexan varios trabajos. De manera consistente se publican muy pocos artículos, con excepciones de los años 2008 (6), 2011 (5), 2013 (6) y 2014 (5). En los primeros 24 años del análisis observamos que se publica un promedio de 1,3 artículos por año.

A partir del 2015 la tendencia cambia. No solo se dispara el promedio de artículos en general, sino que de manera consistente hay una importante producción de artículos que han seguido un camino ascendente. En los cuatro últimos años el promedio de artículos publicados en WoS se elevó a 37,5 artículos por año. Es evidente el incremento extraordinario de la producción intelectual del campo a partir del año 2015, cuando entra en vigor la nueva base de datos ESCI.

Figura 1. Distribución por años de artículos de comunicación indexados en Emerging Sources Citation Index y Social Science Citation Index publicados por instituciones colombianas

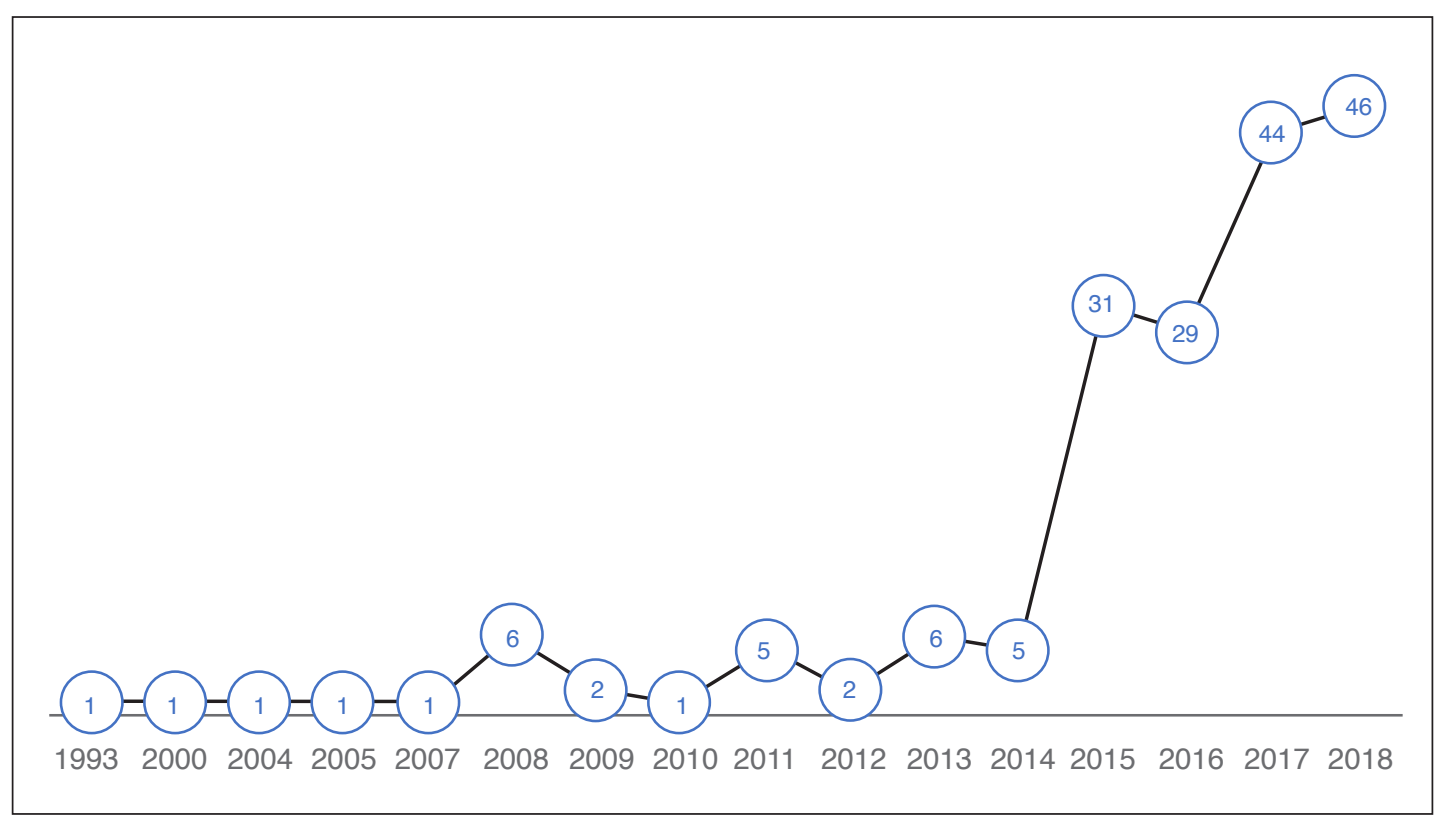

Fuente: elaboración propia

La revista que registra un mayor número de artículos colombianos de comunicación es Palabra Clave publicada por la Universidad de La Sabana. Del grupo de las revistas en el campo de la comunicación, Palabra Clave es también la revista mejor posicionada en el ranking de Scopus en Colombia. En la actualidad ocupa el cuartil 3. A nivel de la indexación de Colciencias en Colombia, publicar en esta revista equivale al denominado grupo top.

En relación con el predominio del idioma, encontramos que la mitad de los artículos se publican en siete revistas, todas ellas iberoamericanas, de las cuales sólo dos están indexadas en los Journal Citation Reports ("El Profesional de la Información" y "Comunicar"). La otra mitad aglutina a 55 revistas donde existen de múltiples nacionalidades, pero en general suelen ser iberoamericanas, publicando artículos en castellano. 
Figura 2. Distribución de trabajos de comunicación colombianos según revistas científicas (más de tres artículos)

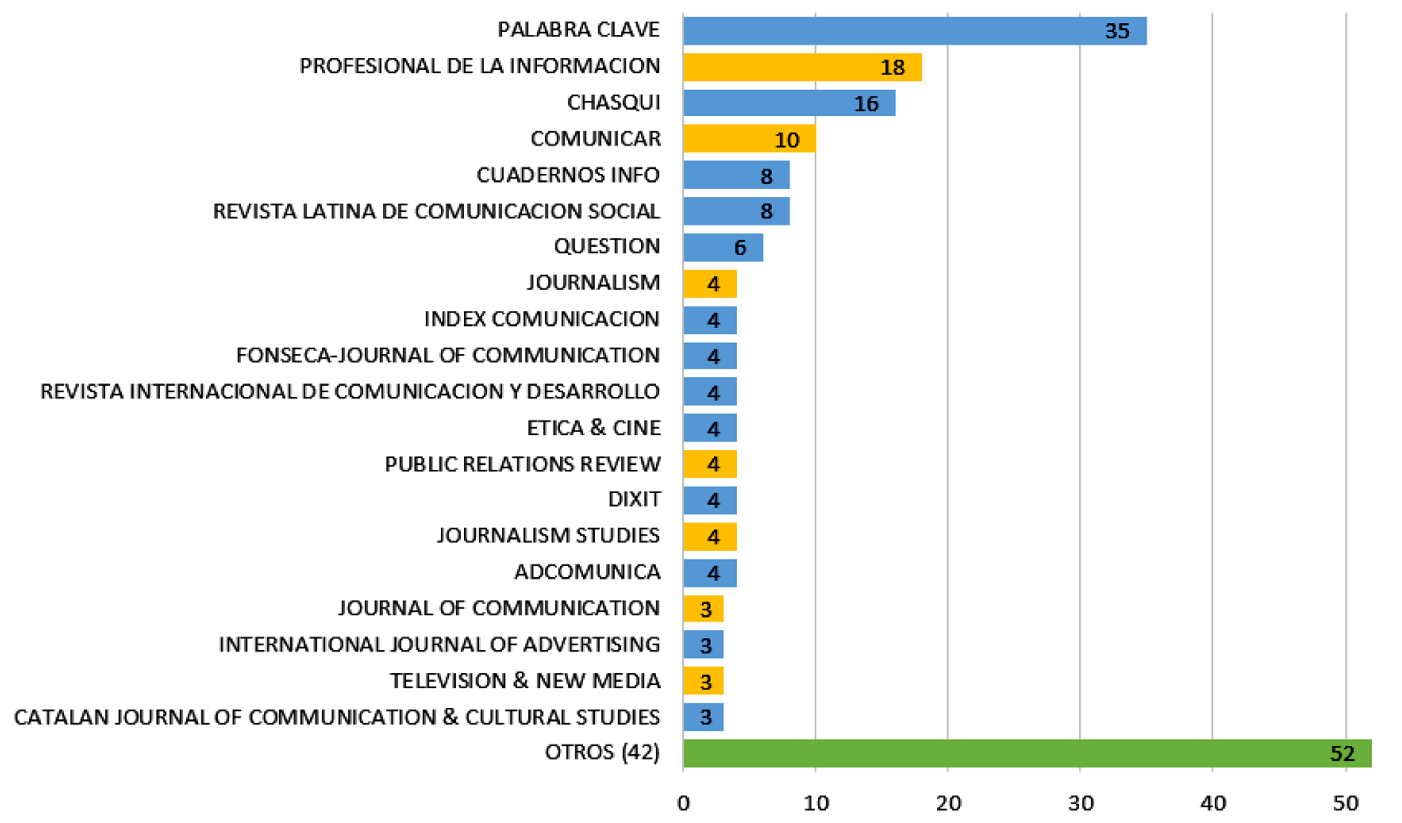

Nota: Se destacan en amarillo las revistas de Social Science Citation Index y en azul las revistas de Emerging Sources Citation Index.

Fuente: elaboración propia

La distribución de artículos en Web of Science según instituciones colombianas (Tabla 2) nos muestra cómo la mayor parte de las instituciones son universidades a excepción del "Observatorio Colombiano de Ciencia y Tecnología". Lo que reafirma que la producción intelectual recae en las universidades y no ya en los centros u observatorios independientes como ocurrió hace años, o como puede ser común en otras disciplinas. El 73\% de los artículos de comunicación colombianos registrados en WoS son publicados por las 8 universidades más productivas, encabezadas por la Universidad del Norte y la Universidad de la Sabana con 29 y 22 trabajos respectivamente.

La hipótesis 1 plantea que habrá mayor productividad y presencia en la base WoS por parte de aquellas instituciones que tienen oferta completa a nivel académico, situación que se podría evidenciar gracias a las tres universidades que lideran la producción de publicaciones en WoS, pues cuentan con oferta completa de la formación académica en el campo que incluye pregrado, maestría y doctorado. La Universidad del Norte, que lidera la lista, fue pionera en Colombia al abrir el primer doctorado en comunicación en Colombia en el 2012. Así mismo, fue pionera en la Costa Caribe al abrir la primera Maestría en Comunicación. La Universidad de la Sabana abrió el segundo doctorado en el campo en mayo del 2017 y ha contado con una oferta variada de postgrados en áreas como Maestría en Comunicación Estratégica y Maestría en Periodismo y Comunicación Digital. Por su parte, la Universidad Javeriana abrió la primera Maestría en Comunicación del país en 1990 y ya tiene aprobado su Doctorado en la misma área. Así mismo, tanto la Universidad Javeriana (Signo y Pensamiento) como la Universidad de la Sabana (Palabra Clave) son las instituciones que han liderado la publicación de revistas en el campo de la comunicación, que han sobresalido en el contexto nacional.

Si tenemos en cuenta la repercusión individual de los trabajos publicados por las universidades nos encontramos que las instituciones universitarias publican trabajos con promedios de citas por debajo de la media internacional de la categoría comunicación. De las 25 universidades colombianas sólo 7 tienen un promedio de impacto superior a la media, mientras que 18 se encuentran en posiciones inferiores a la media mundial. 
Es la Universidad Tecnológica de Bolívar, con tres artículos, la institución con mayor impacto normalizado por categoría en promedio, sin embargo, es quizás más significativo en el caso de la Universidad de la Sabana cuyo promedio de impacto normalizado de sus 26 artículos se sitúa en más del doble del área (2.25). Igualmente, los percentiles de los centros de investigación según el impacto de los trabajos de comunicación publicados se sitúan muy bajos, por debajo del 50 la gran mayoría, con la excepción de la Universidad Tecnológica de Bolívar (3 trabajos) y el Observatorio Colombiano de Ciencia y Tecnología (1 trabajo).

Con respecto a la última hipótesis, se aprecia que, de las 25 instituciones estudiadas, 16 colaboran con otros centros para publicar en comunicación. La institución que destaca por poseer un mayor capital relacional internacional en el área de comunicación es la Universidad del Norte, que colabora con 16 universidades diferentes (12 de ellas colaboraciones internacionales) seguido por la Universidad de la Sabana (7 universidades de las cuales 6 son colaboraciones internacionales). También hay que destacar la alta colaboración de otras instituciones como la Universidad de los Andes (5 centros colaboradores) y la Universidad Pontificia Bolivariana (4 centros colaboradores). Los 22 centros no colombianos con los que las instituciones colombianas colaboran son eminentemente europeos (11 universidades) y estadounidenses ( 9 universidades), donde destacan especialmente las universidades españolas ( 7 universidades).

Tabla 2. Producción e impacto de la investigación en comunicación generada por instituciones colombianas en Web of Science (08/2019)

\begin{tabular}{|c|c|c|c|c|c|c|c|}
\hline \multirow[b]{2}{*}{ INSTITUCIÓN } & \multicolumn{3}{|c|}{ Documentos } & \multirow[b]{2}{*}{ Citas } & \multirow{2}{*}{$\begin{array}{l}\text { Promedio } \\
\text { Citas }\end{array}$} & \multirow[b]{2}{*}{ INC } & \multirow[b]{2}{*}{ Percentil } \\
\hline & $\mathrm{ESCl}$ & $\begin{array}{l}\text { A\&HCl } \\
\text { y SSCl }\end{array}$ & Total & & & & \\
\hline Univ. del Norte & 12 & 17 & 29 & 108 & 3,72 & 1,18 & 48,06 \\
\hline Univ. de La Sabana & 13 & 13 & 26 & 132 & 5,08 & 2,25 & 46,80 \\
\hline Pontificia Univ. Javeriana & 16 & 6 & 22 & 66 & 3,00 & 1,12 & 28,37 \\
\hline Univ. de los Andes & 9 & 10 & 19 & 55 & 2,89 & 0,94 & 38,46 \\
\hline Univ. de Medellín & 13 & 3 & 16 & 19 & 1,19 & 0,14 & 16,49 \\
\hline Univ. de Antioquia & 6 & 8 & 14 & 35 & 2,50 & 0,46 & 32,68 \\
\hline Univ. Pontificia Bolivariana & 11 & 3 & 14 & 13 & 0,93 & 0,35 & 23,62 \\
\hline Univ. del Rosario & 11 & 2 & 13 & 38 & 2,92 & 0,55 & 24,89 \\
\hline Univ. del Valle & 3 & 3 & 6 & 21 & 3,50 & 1,04 & 43,33 \\
\hline Univ. EAFIT & 5 & 1 & 6 & 1 & 0,17 & 0,06 & 7,33 \\
\hline Univ. del Tolima & 5 & 0 & 5 & 0 & 0,00 & 0,00 & 0,00 \\
\hline Univ. Nacional de Colombia & 4 & 1 & 5 & 1 & 0,20 & $\overline{0,04}$ & 7,42 \\
\hline Univ. Externado & 3 & 1 & 4 & 9 & 2,25 & 0,52 & 36,45 \\
\hline Univ. de Cartagena & 3 & 0 & 3 & 0 & 0,00 & 0,00 & 0,00 \\
\hline Univ. Tecnológica de Bolívar & 1 & 2 & 3 & 11 & 3,67 & 2,60 & 61,91 \\
\hline Univ. Autónoma de Occidente & 0 & 2 & 2 & 0 & 0,00 & 0,00 & 0,00 \\
\hline Univ. Aut. Latinoamericana & 2 & 0 & 2 & 0 & 0,00 & 0,00 & 0,00 \\
\hline Univ. de la Costa & 1 & 1 & 2 & 5 & 2,50 & 1,77 & 45,89 \\
\hline Univ. del Cauca & 1 & 1 & 2 & 1 & 0,50 & 0,68 & 34,21 \\
\hline Univ. Industrial de Santander & 1 & 1 & 2 & 3 & 1,50 & 0,17 & 26,08 \\
\hline Univ. Tecnológica de Pereira & 2 & 0 & 2 & 0 & 0,00 & 0,00 & 0,00 \\
\hline Obs. Colomb. de Cienc. y Tecn. & 0 & 1 & 1 & 8 & 8,00 & 2,52 & 90,76 \\
\hline Univ. de Caldas & 1 & 0 & 1 & 0 & 0,00 & 0,00 & 0,00 \\
\hline Univ. de La Salle & 1 & 0 & 1 & 0 & 0,00 & 0,00 & 0,00 \\
\hline Univ. ICESI & 0 & 1 & 1 & 3 & 3,00 & 0,23 & 46,79 \\
\hline
\end{tabular}

Nota: INC= Impacto Normalizado por Categoría

Fuente: elaboración propia 
Las universidades que más colaboran son a su vez aquellas que más producen y reciben un impacto normalizado mayor, destacando la Universidad del Norte. De manera que se confirma la tendencia de que las instituciones con mayor capital relacional internacional tienen mayores opciones de publicar en la base de datos WoS.

Se observan cuatro clústeres principales. El mayor es el encabezado por la Universidad del Norte (8 nodos) que aglutina a la Universidad del Externado y a la Universidad Iberoamericana junto con seis universidades extranjeras. En segundo lugar, nos encontraríamos un grupo poco cohesionado liderado por la Universidad de Medellín que cuenta con cuatro universidades colombianas y cuatro universidades extranjeras ( 2 españolas, 1 mexicana y una estadounidense). Le siguen dos grupos por tamaño, el liderado por la Universidad de La Sabana junto a 5 universidades internacionales y el de las Universidades de los Andes y la Pontificia Bolivariana, cuya esencia es principalmente colombiana.

Figura 3. Red de colaboración interinstitucional. Investigación colombiana en Web of Science

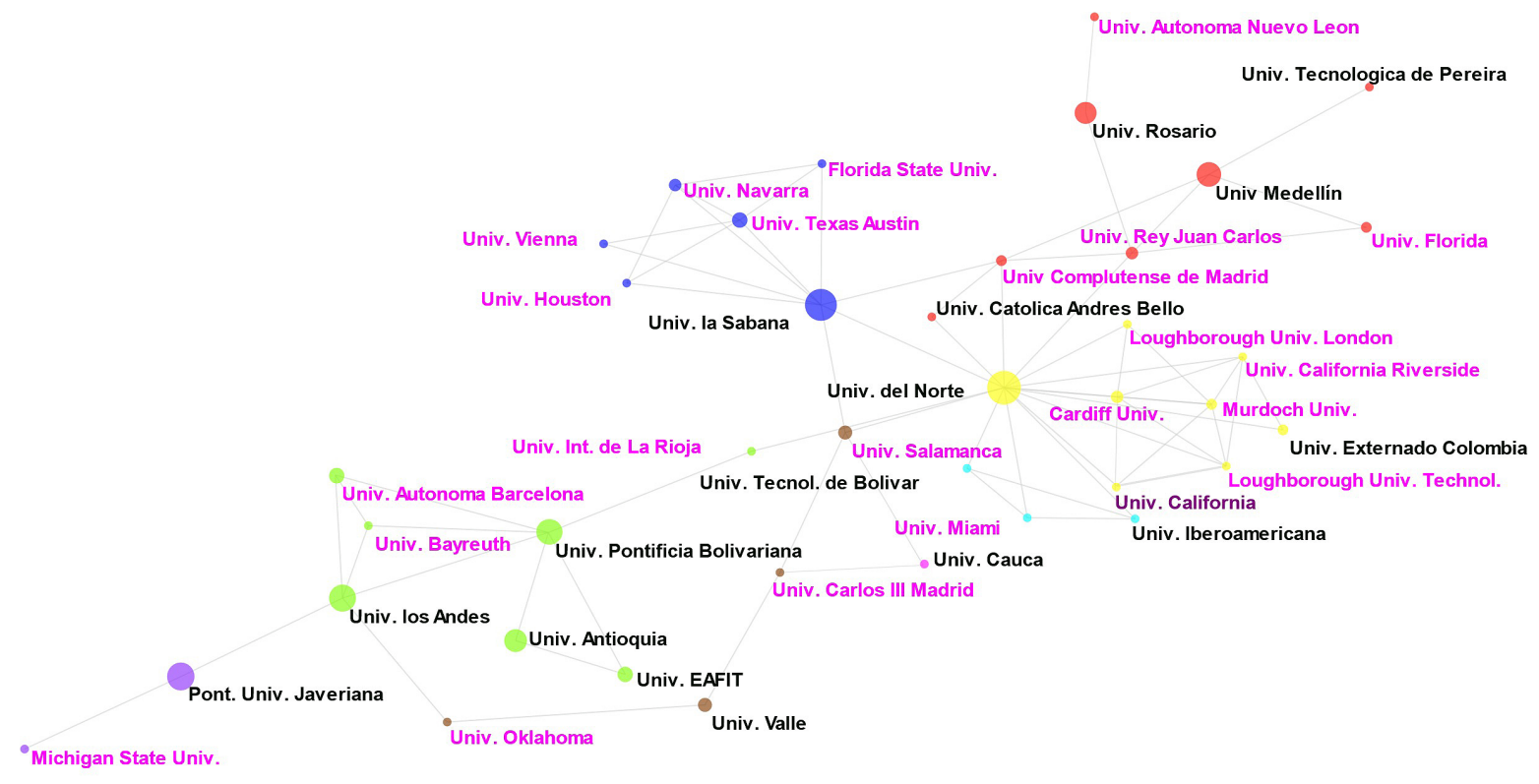

Fuente: elaboración propia

\section{Discusión y conclusiones}

La presente investigación se trazó como objetivo establecer cómo ha sido la producción y circulación de conocimiento de la investigación colombiana en la base de datos de mayor reconocimiento internacional como lo es Web of Science (WoS). Así mismo, identificar las redes internacionales de investigación en comunicación en Colombia usando la coautoría de las publicaciones en las revistas indexada en WoS. Los hallazgos demuestran que la investigación colombiana en comunicación ha ganado mayor presencia en esta base de datos y que ha recorrido un largo camino hasta lograr un posicionamiento importante.

La apertura de la base de datos WoS con la creación del "Emerging Sources Citation Index" ha permitido un crecimiento exponencial de publicaciones de autores afiliados a la academia colombiana en esta base hegemónica. En 24 años (1993 - 2014) se produjeron 32 artículos, a razón de 1.3 artículo por año en promedio. Con la creación de ESCI en el 2015, 31 artículos se publicaron tan solo en ese año, equiparando la producción intelectual del último cuarto de siglo. En los próximos 3 años alcanzaría los 150 artículos, casi quintuplicando lo realizado los primeros 24 años. Desde la primera publicación de Martin-Barbero en 1993 en el Journal of Communication, 
la presencia en esta base de datos de la investigación colombiana en comunicación ha dado un salto cualitativo.

Seis revistas han liderado la publicación de conocimiento en comunicación de autores vinculados a la academia colombiana, tres de ellas son españolas: "El Profesional de la Información", "Comunicar" y "Revista Latina de comunicación Social". Una es chilena "Cuadernos.info" y la otra ecuatoriana "Chasqui". La única colombiana de este grupo que aglutina la mayor cantidad de artículos es "Palabra Clave". Todas estas revistas fueron incorporadas en la ampliación de WoS del año 2008 o en la posterior creación de ESCI (2015).

Sobresale entonces la revista "Palabra Clave", que es la única revista colombiana de comunicación indexada en Web of Science Core Collection, por lo que es de facto la revista portavoz de la investigación colombiana para el mundo. El 23,4\% del total de artículos en comunicación estudiados fueron publicados en "Palabra Clave" o lo que equivale a más de 1 de cada 5 artículos. Se ha consolidado entonces como la publicación con más artículos de autores colombianos en esta base de datos internacional en el último lustro.

Dado que las redes de colaboración y coautorías entre investigadores son también una fuente medible de capital académico (Coccia \& Bozeman, 2016), y que a mayor capital relacional a nivel internacional mayores son las posibilidades de publicar en revista de alta indexación (Demeter, 2019), los hallazgos de este estudio ofrecen evidencia empírica de este planteamiento teórico. En efecto, las cinco universidades que más publicaciones tienen en WoS sobresalen por ser aquellas que mayor número de relaciones internacionales poseen.

Estos hallazgos guardan concordancia con la investigación adelantada por Ávila-Toscano, Marenco-Escuderos y Romero Pérez (2019), en donde analizaron las propiedades estructurales en redes de coautoría y colaboración institucional en ciencias sociales, a partir de los artículos publicados entre 2006 y 2015 por 3804 autores de siete disciplinas vinculados a grupos de investigación registrados en el Sistema Científico Nacional de Colombia (ScienTI-COL). Los investigadores encontraron que en el área de Periodismo la Universidad del Norte destacaba por su red de colaboración internacional en la producción científica, concluyendo que: "la red se sostiene por la participación de la Universidad del Norte y la Complutense de Madrid, sin ellas incluso el flujo de interacciones colapsaría perdiéndose las conexiones entre organismos de Colombia y el exterior" (Ávila Toscano et al., 2019, p. 222).

Se observa una correspondencia con la tendencia internacional según la cual la co-autoría con múltiples instituciones ha crecido de manera exponencial en las ciencias. El informe del Science and Technology Observatory-OST (2019) señala que la tendencia científica más habitual es la colaboración entre instituciones para realizar investigación mientras que se reduce el número de publicaciones con una sola institución. Entre los años 2000 y 2016, el número de colaboraciones entre instituciones se triplicaron, y "el número internacional de co-publicaciones virtualmente se cuadruplicó" (p. 26). Estos datos se completan con el informe de The Royal Society (2009), que enfatiza que la colaboración dentro de la Unión Europea tiende a tener un menor impacto que las publicaciones trasatlánticas en las diferentes disciplinas (p. 6).

Cabe destacar que las universidades que sobresalen tanto en producción intelectual en WoS como en mayor número de redes internacionales sobresalen por tener una oferta de formación académica completa. Ambas han sido pioneras en Colombia con respecto a la oferta de postgrado del más alto nivel. De una parte, la Universidad del Norte fue pionera en Colombia al abrir el primer doctorado en comunicación en el 2012. Por su parte, la Universidad de la Sabana abrió el segundo doctorado en Colombia en el 2017. La Universidad Javeriana y la Universidad de Medellín cuentan también con doctorados en comunicación recientemente aprobados. Ciertamente la oferta de formación de 
postgrado a nivel de investigación demanda un esfuerzo importante tanto en cualificar la investigación como en hacerla más visible a nivel internacional.

Este estudio no está exento de limitaciones. En primer lugar, lo reportado aquí se limita exclusivamente a la producción científica publicada en una sola base de datos, WoS, hasta el año 2018. Si bien es hoy considerada la más usada por las grandes instituciones científicas y académicas internacionales, no puede tomarse como único criterio de calidad académica. Así mismo, existen otras revistas indexadas de nivel que no aparecen en esta indexación. Otro tipo de publicaciones tales como informes, reportes y diversos textos presentados en conferencias y eventos académicos también dan cuenta de una producción científica de gran nivel, aunque no aparece en este tipo de bases de datos. Otras bases de datos que han surgido como respuesta al carácter elitista de WoS y la necesidad de publicar en español como Latindex, Dialnet, DOAJ, REDIB no fueron incluidas, sin embargo, sí han sido objeto de análisis de otras publicaciones (González, Repiso \& Arroyave, 2020). Para estudios futuros queda la propuesta de efectuar un análisis comparativo con la otra base de datos Scopus.

La presente investigación contribuye al acervo teórico sobre la producción intelectual del campo de la comunicación en las principales bases de datos internacionales. Explora un tema importante que hasta ahora ha sido poco estudiado en el país. Así mismo, contribuye con un aporte empírico que permite continuar una línea de investigación, que en el campo internacional ya se ha ido consolidando (Demeter, 2017b, 2018, 2019).

En términos teóricos, si bien siguiendo a Bourdieu (1988, 1988, 2004), el campo académico puede ser considerado como un juego regido por ciertas reglas que han sido establecidas por jugadores que cuentan con un alto capital, dichas reglas están en constante cuestionamiento, son fluidas y cambiantes. La "des-occidentalización" de la investigación es un tema de debate actual (Waisbord \& Mellado, 2014; Ng, White \& Saha, 2020), así como la necesidad de incluir nuevas voces (Chakravartty, 2020; Chakravartty et al., 2018; Hanitzsch, 2019) y de permitir a otras epistemologías, formas de investigación y estructuras de investigación tener cabida en este juego en condiciones de igualdad (Mignolo, 2010). La creación de ESCI ha permitido que otras revistas hagan carrera en la plataforma WoS y que den los pasos iniciales para romper la hegemonía de las publicaciones tradicionales y se abran caminos a nuevas voces que permitan mayor diversidad.

\section{Bibliografía}

Albuquerque, A., Moreira de Olivera, T., Alves dos Santos, M., \& Firmo de Albuquerque, S. (2020). Structural limits to the De-Westernization of the communication field: The editorial board in Clarivate's JCR System. Communication, Culture \& Critique 13, 185-203.

Alejo Montes, F. J. (2007). La docencia en la Universidad de Salamanca en el Siglo de Oro. Salamanca: Ediciones Universidad de Salamanca.

Altbach, P. G. (2007). Peripheries and Centers: Universities in Developing Countries. Higher Education Management and Policy, 19 (2): 111-134. https://doi.org/10.1787/hemp-v19-art13-en

Anzola, P. y Cooper., P. (1985). La investigación en comunicación social en Colombia. Bogotá: DECSCO, ACICS.

Ávila-Toscano, J. H., Marenco-Escuderos. A.D. \& Romero-Pérez, I. K. (2019). Redes de cooperación entre autores e instituciones en Ciencias Sociales dentro del modelo científico colombiano: comparación por género y área del conocimiento. Revista General de Información y Documentación, 29 (1), 209-227. https://doi.org/10.5209/rgid.64545

Barranquero, A., Arcila, C., \& Arroyave, J. (2017). Manual De Teoría De La Comunicación II. Pensamiento Latinoamericano. Barranquilla, Colombia: Editorial Universidad del Norte: 
Bourdieu, P. (1988). Homo academicus. Stanford, CA: Stanford University Press.

Bourdieu, P. (1998). Practical reason: On the theory of action. Stanford, CA: Stanford University Press.

Bourdieu, P. (2004). Science of Science and reflexivity. Cambridge, UK: Polity Press.

Beigel, F. (2013). David y Goliath. El sistema académico mundial y las perspectivas del conocimiento producido en la periferia. Pensamiento Universitario 15, 1-18.

Beigel, F. (2014). Publishing from the periphery: Structural heterogeneity and segmented circuits. The evaluation of scientific publications for tenure in Argentina's CONICET. Current Sociolog, 62(5), 743-765. https://doi.org/10.1177/0011392114533977

Beigel, F. (2016). Científicos Periféricos, entre Ariel y Calibán. Saberes Institucionales y Circuitos de Consagración en Argentina: Las Publicaciones de los Investigadores del CONICET. Revista Dados, 59(4), 215-255. https://doi.org/10.1590/001152582017136

Chakravartty,P., (2020). \#CommunicationSoWhite in the age of ultra-nationalism. Communication, Culture \& Critique 13, 270-274.

Chakravartty,P., Kuo,R., Grubbs,V., \& McIlwain, C. (2018). \#CommunicationSoWhite Journal of Communication 68 (2), 254-266 doi: https://doi.org/10.1093/joc/jqy003

Coccia, M. \& Bozeman, B. (2016). Allometric models to measure and analyze the evolution of international research collaboration. Scientomeytrics, 108(3), 1065.1084. https://10.1007/s11192016-2027-x

Colciencias (2018). Modelo de medición de grupos de investigación, desarrollo tecnológico o de innovación y de reconocimiento de investigadores del sistema nacional de ciencias, tecnología e innovación, 2018. Departamento Administrativo de Ciencia, Tecnología e Innovación-Colciencias. Bogotá: Colombia.

Demeter, M. (2019). So Far, Yet So Close: International Career Paths of Communication Scholars from the Global South. International Journal of Communication 13, 578-602.

Demeter, M. (2018). The winner takes it all: International inequality in communication and media studies today. Journalism and Mass Communication Quarterly. 96 (1), 37-59. https://doi. org/10.1177/1077699018792270

Demeter, M. (2017a). Author productivity index: Without distortions. Science and Engineering Ethic. 24, 1661-1663. https://doi:10.1007/s11948-017-9954-7

Demeter, M. (2017b). The core-periphery problem in communication research: A network analysis of leading publication. Publishing Research Quarterly 33(4), 402-420. https://doi:10.1007/ s12109-017- 9535-2

Downing, J. (1996). Internationalizing media theory: Transition, power, culture. London, UK: SAGE Publications.

Fernández, A., Ferrandiz, E., \& León, M. D. (2016). Proximity dimensions and scientific collaboration among academic institutions in Europe: The closer, the better. Scientometrics, 106, 1073-1092. https://doi:10.1007/s11192-015-1819-8

Flores, C. V., Palavecino, R. A. \& Montejano, G. (2013). Indicadores de capital relacional en la investigación Universitaria. 5to Simposio Internacional de Investigación "Interdisciplinariedad, Multidisciplinariedad y/o Transdisciplinariedad: en la búsqueda de respuestas desde las 
experiencias de Investigación”. San Salvador de Jujuy, agosto. Universidad Católica de Santiago del, (August 2013), 978-987.

Freeman, L. (2006). The Development of Social Network Analysis: A study in the Sociology of Science. Vancouver: Empirical Press.

Fischman, G., Alperin, J. P., \& Willisnky, J. (2010). Visibility and quality in Spanish-language: Latin American scholarly publishing. Information Technologies and Intyernational Development 6(4), 1-21.

Funes, C. , Heredia, C. \& Suárez, V. (2011). Las revistas cientificas latinoamericanas en el ISI Web of Science: una opción para académicos e investigadores. Serie Bibliotecología y Gestión de Información 65, mayo 11. Universidad Tecnológica Metropitibana, Santiago: Chile.

González-Pardo, R., Repiso, R., \& Arroyave, J. (2020). Revistas iberoamericanas de comunicación a través de las bases de datos Latindex, Dialnet, DOAJ, Scopus, AHCI, SSCI, REDIB, MIAR, ESCI y Google Scholar Metrics. Revista Española de Documentación Cientifica (Próximo a publicarse).

Goyanes, M. (2020). Editorial boards in communication sciences journals: Plurality or standardization. The International Communication Gazzette 82(4), 342-364.

Hanitzsch, T. (2019). Journalism studies still needs to fix Western bias. Journalism, 20(1), 214-217.

Lucas, C. J. (1994). American Higher Education. A History. New York: St. Martin's Press.

Martín-Barbero, J., \& Rey. G. (1999). La formación del campo de estudios de comunicación en Colombia. Revista de Estudios Sociales 4, 54- 68.

Mignolo, W. (2010). Desobediencia epistémica: retórica de la modernidad, lógica de la colonialidad y gramática de la descolonialidad. Buenos Aires: Ediciones del Signo.

Mosbah-Natanson, S., \& Gingras, Y. (2013). The globalization of social sciences? Evidence from a quantitative analysis of 30 years of production, collaboration and citations in the social. Current Sociology, 0 (0), 1-20. https://doi.org/10.1177/0011392113498866

Ng, E., Costley White, K. \& Saha, A. (2020). \#CommunicationSoWhite: Race and power in the academy and beyond. Communication, Culture \& Critique 13,143-151.

Noble, K. A. (1994). Changing doctoral degrees: An international perspective. The Society for Research into Higher Education Series. Bristol, PA: Open University Press.

Ortiz, R. (2009). La supremacía del inglés en las ciencias sociales. Buenos Aires: Siglo XXI.

OST- Science and Technology Observatory (2019). Dynamics of scientific production in the world, in Europe and in France, 2000-2016. París: Hcéres.

Palomero Pescador, J. E. (2010). Los índices de impacto, una mirada crítica. Revista Interuniversitaria de Formación del Profesorado (RIFOP), octubre. http://bit.ly/37kFcLr

Pereira, J. M. (2018). La investigación en comunicación en Colombia: Síntesis (in)acabada de una travesía. En Crovi, D. \& Trejo, R. (Coords.), Tejiendo nuestra historia. Investigación de la comunicación en América Latina. (pp. 177-201). Ciudad de México: UNAM.

Post, D., Stambach, A., Ginsburg, M., Hannum, E., Benavot, A. \& Bjork, Ch. (2013). Los rankings académicos. Archivos Analiticos de Políticas Educativas, 21 (19), https://doi. org/10.14507/epaa.v21n19.2013

Ready, D. (2014). From academic freedom to academic capitalism. Discover Society, February, http://bit.ly/2O99RnD 
Repiso, R. y Torres-Salinas, D. (2016). Características e implicaciones de la base de datos Emerging Sources Citation Index (Thomson Reuters): las revistas en estado transitorio. Anuario ThinkEPI, 10, 234-236.

Rogers, E. M. (1994). A history of communication study: A biographical approach. New York: The Free Press.

Roncallo-Dow, S., Uribe-Jongbloed, E., y Calderón, I. (2013). La investigación en comunicación. Límites y limitantes de conocimiento. Co-Herencia 10(18), 161-187.

San Fabián, J. L. (2020). El reconocimiento de la actividad investigadora universitaria como mecanismo de regulación del mercado académico. Márgenes, Revista de Educación de la Universidad de Málaga 1 (1), 23-44. http://dx.doi.org/10.24310/mgnmar.v1i1.7208

Sanz Menéndez, L. (2003). Análisis de redes sociales: o cómo representar las estructuras sociales subyacentes. Apuntes de Ciencia y Tecnología 7, 21-29.

Schmoch, U., \& Schubert, T. (2008). Are international co-publications an indicator for quality of scientific research? Scientometrics, 74, 361-377.

Scott, J. (1992). Social Network Analysis. Newbury Park Ca: Sage.

Teodorescu, D., \& Tudorel, A. (2011). The growth of international collaboration in EastEuropean scholarly communities: A bibliometric analysis of journal articles published between 1989 and 2009. Scientometrics 89, 711-722.

The Royal Society (2011). Knowledge, Networks and Nations: Global scientific collaboration in the 21st century. London: The Royal Society.

Thelin, J. R. (2004). A History of American Higher Education. Baltimore: The Johns Hopkins University Press.

Torres-Salinas, D., Robinson-García, N., Herrera-Viedma, E., \& Jiménez-Contreras, E. (2018). Consideraciones metodológicas sobre uso del impacto normalizado en convocatorias Severo Ochoa y María de Maetzu. El Profesional de La Información 27(2), 367. https://doi.org/10.3145/epi.2018.mar.15

UNESCO (2005). Unesco Science Report: Paris, UNESCO.

UNESCO (2010). Unesco Science Report: The current status of science around the world. Paris, UNESCO.

UNESCO (2015). Unesco Science Report: Toward 2030. Paris, UNESCO.

Valderrama, C. (2009). La investigación en medios de comunicación en Colombia (1980-2009). Revista Nómadas 31, 262-276.

Valderrama, C., S. Rojas y V. González. (2011). Medios de comunicación en Colombia. Treinta años de investigación y reflexión. Bogotá: Universidad Central.

Waisbord, S. \& Mellado, C. (2014). De-westerninzing communication studies: A reassesment. Communicatgion Theory 24(4), 361-372.

Weinberg, G. (1997). “Ilustración” y Educación Superior en Hispanoamérica Siglo XVIII. Buenos Aires: Academia Nacional de Educación.

Zdenek, R. (2018). Editorial board self-publishing rates in Czech economic journals. Science and Engineering Ethics 24(2),669-682. https://10.1007/s11948-017-9922-2. 